MITH-97/3

\title{
Fine-tuning the low-energy physics
}

\author{
She-Sheng Xue \\ Dipartimento di Fisica, Universitá di Milano \\ INFN - Sezione di Milano, Via Celoria 16, Milan, Italy
}

\begin{abstract}
In this article we discuss the problem of the extreme fine-tuning necessary to achieve the top quark mass scale $m_{t}$ within the dynamical symmetry breaking of the phenomenological $t \bar{t}$ condensate model. Inspired by the vector-like phenomenon of chiral gauge theories at short distances, we postulate that the $W^{ \pm}$-gauge bosons possess a vector-like gauge coupling and contribute to the gap-equations of quark self-energy functions in the high-energy region. We find that the gap-equations of different charge sectors are coupled, and as a result the unnatural fine-tuning is overcome and the ratio of the top and bottom masses is related to the electric charge: $\left(\frac{m_{b}}{m_{t}}\right)^{2} \simeq \frac{\alpha}{3 \pi}$, provided the quadratic divergence is removed by setting the four-fermion coupling $G=4+o\left(\frac{m_{b}^{2}}{m_{t}^{2}}\right)$.
\end{abstract}

December, 1996

PACS 11.15Ha, 11.30.Rd, 11.30.Qc

a) E-mail address: xue@milano.infn.it 


\section{Introduction}

It is well known that the dynamical symmetry breaking of the NambuJona Lasinio (NJL) type [1] meets the severe problem of extreme fine-tuning. Indeed the gap equation obeyed by the mass gap turns out to be quadratically divergent $\left(O\left(\Lambda^{2}\right)\right)$, and in the absence of a symmetry that protects the mass gap from such divergence, the only way out is to tune the NJL coupling to a critical value with the incredible precision of $\left(\frac{m}{\Lambda}\right)^{2}$. And this is clearly very unnatural. Analogously, the vacuum expectation value of the elementary scalar field envisaged in the Higgs mechanism is also plagued by quadratic divergences, stemming from radiative corrections, and a similar fine-tuning of the $\phi^{4}$-potential is needed in order to eliminate the quadratic divergences. Supersymmetry with its implied cancellation of the quadratic divergences between the bosonic and fermionic sectors of the theory thus becomes a very strong theoretical candidate to cure such problem. This suggests that the solution of the problem implies a highly non-trivial structure of the theory in the high-energy region.

In this article we wish to show that there is another solution, which is much more economical in terms of particles and interactions. In section 2 , we focus on the third quark family to carefully describe fine-tuning in the context of dynamical symmetry breaking of the phenomenological $t \bar{t}$ condensate model. In section 3, we discuss our motivation and hypothesis of the $W^{ \pm}$ gauge boson's coupling and its contribution to the Schwinger-Dyson equations of fermion masses. Such coupled Schwinger-Dyson equations are solved in section 4. The discussions of how the problem of fine-tuning is evaded and some remarks are presented in the last section.

\section{The problem of fine-tuning}

In order to explicitly show how we obtain the low-energy weak scale by dynamical symmetry breakings, we consider the top-condensate BHL-model 22 for the third quark generation of the top and bottom quarks. This model is phenomenologicallyt given by the lagrangian,

$$
L=L_{\text {standard }}+G_{\circ} \bar{\Psi}_{L}^{i a}(x) \cdot t_{R}^{a}(x) \bar{t}_{R}^{b}(x) \cdot \Psi_{L}^{i b}(x),
$$

where $\Psi_{L}^{i}(x)$ is the left-handed weak doublet and $a, b$ are color indices and $G_{\circ}$ is the four-fermion coupling, due to some underlying physics at the cutoff

\footnotetext{
${ }^{1}$ In ref.[3], we have shown that the solution of only one very massive quark is energetically favourable.
} 
scale $\Lambda$. When the four-fermion coupling and the electric coupling (charge) are larger than certain critical values, this model develops the phenomenon of spontaneous symmetry breaking and a non-zero fermion self-energy function $\Sigma_{t}(p)$ of the top quark emerges. To illustrate fine-tuning needed to obtain $\Sigma_{t}(p) \ll \Lambda$ in the low-energy limit, first we briefly summarize some basic features about the Schwinger-Dyson approach that were discussed in the refs. 田.

Owing to its purely left-handed gauge coupling to fermions, the $W$-boson does not contribute to the Schwinger-Dyson equations. Note that we ignore the $Z$-boson's contribution for its perturbative character and the QCDcontribution for its confining property. We only take the QED and the four-fermion interaction into account. In the Landau gauge, the fermion self-energy functions $\Sigma_{t, b}(p)$ for the top and bottom quark satisfy,

$$
\begin{aligned}
& \Sigma_{t}\left(p^{2}\right)=m-\frac{G_{\circ}}{2}\langle\bar{t} t\rangle+3 Q^{2} \int_{p^{\prime}}^{\Lambda} \frac{1}{\left(p-p^{\prime}\right)^{2}} \frac{\Sigma_{t}\left(p^{\prime 2}\right)}{p^{2}+\Sigma_{t}^{2}\left(p^{\prime 2}\right)} \\
& \Sigma_{b}\left(p^{2}\right)=m+3 \frac{Q^{2}}{4} \int_{p^{\prime}}^{\Lambda} \frac{1}{\left(p-p^{\prime}\right)^{2}} \frac{\Sigma_{b}\left(p^{\prime 2}\right)}{p^{\prime 2}+\Sigma_{b}^{2}\left(p^{\prime 2}\right)}
\end{aligned}
$$

where $m$ is the bare mass added to the lagrangian (1), $Q=\frac{2}{3} e$ is the bare charge of the top quark at the cutoff. After performing the angular integration and changing variables to $x=p^{2}$, eqs.(2, 3) can be converted to the following boundary value problems:

$$
\begin{aligned}
\frac{d}{d x}\left(x^{2} \Sigma_{t}^{\prime}(x)\right)+\frac{\alpha_{t}}{4 \alpha_{c}} \frac{x}{x+\Sigma_{t}^{2}(x)} \Sigma_{t}(x) & =0, \\
(1+g) \Lambda^{2} \Sigma_{t}^{\prime}\left(\Lambda^{2}\right)+\Sigma_{t}\left(\Lambda^{2}\right) & =m,
\end{aligned}
$$

and

$$
\begin{aligned}
\frac{d}{d x}\left(x^{2} \Sigma_{b}^{\prime}(x)\right)+\frac{\alpha_{t}}{16 \alpha_{c}} \frac{x}{x+\Sigma_{b}^{2}(x)} \Sigma_{b}(x) & =0 \\
\Lambda^{2} \Sigma_{b}^{\prime}\left(\Lambda^{2}\right)+\Sigma_{b}\left(\Lambda^{2}\right) & =m
\end{aligned}
$$

where the notations are:

$$
\alpha_{t}=\frac{Q^{2}(\Lambda)}{4 \pi} ; \quad \alpha_{c}=\frac{\pi}{3} ; \quad g=\frac{N_{c} G_{\circ} \Lambda^{2}}{2 \pi^{2}} \frac{\alpha_{c}}{\alpha_{t}} ; \quad\langle\bar{t} t\rangle=\Lambda^{4} \frac{\Sigma_{t}^{\prime}\left(\Lambda^{2}\right)}{3 \pi \alpha_{t}} .
$$


Note that the bare mass $m$ and the four-fermion coupling $g$ enter the gap equation through the boundary condition only. For $x \gg 1$, the nonlinearity present in the boundary value problem can be neglected, the solutions for the self-energy functions in the ultraviolet region are given,

$$
\begin{aligned}
& \Sigma_{t}(x)=\frac{A_{t} \mu^{2}}{\sqrt{x}} \sinh \left(\frac{1}{2} \sqrt{1-\frac{\alpha_{t}}{\alpha_{c}}} \ln \left(\frac{x}{\mu^{2}}\right)\right) ; \\
& \Sigma_{b}(x)=\frac{A_{b} \mu^{2}}{\sqrt{x}} \sinh \left(\frac{1}{2} \sqrt{1-\frac{\alpha_{t}}{4 \alpha_{c}}} \ln \left(\frac{x}{\mu^{2}}\right)\right),
\end{aligned}
$$

where $A_{t, b}$ are finite constants, that should be related to the Yukawa couplings of the top and bottom quarks, and $\mu$ some infrared scale. The A's and $\mu$, in principle, are determined by the boundary conditions at both the infrared limit and the ultraviolet limit. Note that solutions (9) and (10) hold in the ultraviolet limit, thus we are only allowed to enforce the ultraviolet boundary conditions (5,7). Using these boundary conditions, we obtain the gap-equations:

$$
\begin{aligned}
& m=\frac{A_{t} \mu^{2}}{2 \Lambda}\left[(1-g) \sinh \theta+(1+g) \sqrt{1-\frac{\alpha_{t}}{\alpha_{c}}} \cosh \theta\right] ; \\
& m=\frac{A_{b} \mu^{2}}{2 \Lambda}\left[\sinh \theta^{\prime}+\sqrt{1-\frac{\alpha_{t}}{4 \alpha_{c}}} \cosh \theta^{\prime}\right],
\end{aligned}
$$

where

$$
\theta=\frac{1}{2} \sqrt{1-\frac{\alpha_{t}}{\alpha_{c}}} \ln \left(\frac{\Lambda^{2}}{\mu^{2}}\right),
$$

and $\theta^{\prime}$ is defined as eq.(13) with substitution $\alpha_{t} \rightarrow \frac{\alpha_{t}}{4}$. The gap-equations (11,12) for the top and bottom quarks can be expressed as

$$
\begin{aligned}
m & =\frac{A_{t} \mu}{4}\left(\frac{\mu}{\Lambda}\right)^{1-\sqrt{1-\frac{\alpha_{t}}{\alpha_{c}}}}\left[1-g+(1+g) \sqrt{1-\frac{\alpha_{t}}{\alpha_{c}}}\right] \\
& +\frac{A_{t} \mu}{4}\left(\frac{\mu}{\Lambda}\right)^{1+\sqrt{1-\frac{\alpha_{t}}{\alpha_{c}}}}\left[(1+g) \sqrt{1-\frac{\alpha_{t}}{\alpha_{c}}}-(1-g)\right] . \\
m & =\frac{A_{b} \mu}{4}\left(\frac{\mu}{\Lambda}\right)^{1-\sqrt{1-\frac{\alpha_{t}}{4 \alpha_{c}}}}\left[1+\sqrt{1-\frac{\alpha_{t}}{4 \alpha_{c}}}\right] \\
& +\frac{A_{b} \mu}{4}\left(\frac{\mu}{\Lambda}\right)^{1+\sqrt{1-\frac{\alpha_{t}}{4 \alpha_{c}}}}\left[\sqrt{1-\frac{\alpha_{t}}{4 \alpha_{c}}}-1\right] .
\end{aligned}
$$


These are the gap-equations that minimize the vacuum energy of the theory. Given $g$ and $\alpha_{t}$, these equations establish the relationship between the cutoff $\Lambda$, the infrared limit $\mu$ and the $A$ 's.

In the case of explicit symmetry breaking $(m \neq 0)$, we have $(\mu \ll \Lambda)$

$$
\begin{aligned}
& m=\frac{A_{t} \mu}{4}\left(\frac{\mu}{\Lambda}\right)^{\frac{\alpha_{t}}{2 \alpha_{c}}}\left[2-(1+g) \frac{\alpha_{t}}{2 \alpha_{c}}\right], \\
& m=\frac{A_{b} \mu}{4}\left(\frac{\mu}{\Lambda}\right)^{\frac{\alpha_{t}}{8 \alpha_{c}}}\left[2-\frac{\alpha_{t}}{8 \alpha_{c}}\right] .
\end{aligned}
$$

The top quark mass $m_{t}=A_{t} \mu$ and the bottom quark mass $m_{b}=A_{b} \mu$ are related to the finite bare mass $m$, their scaling behaviours in the infrared limit $\left(\frac{\mu}{\Lambda} \ll 1\right)$ are given by the equations (16,17). No fine-tuning of the couplings $g$ and $\alpha_{t}$ is needed.

In the chiral limit $(m=0)$, i.e. when no explicit symmetry breaking occurs, we study how the low-energy limit $(\mu \ll \Lambda)$ is obtained after the spontaneous symmetry breaking. From the gap-equation (14) one finds for large values of the four-fermion coupling $g$ and QED coupling $\alpha_{t}$,

$$
A_{t} \neq 0, \quad\left(\frac{\mu}{\Lambda}\right)^{2}=\left[\frac{1-g+(1+g) \sqrt{1-\frac{\alpha_{t}}{\alpha_{c}}}}{2(1-g)}\right]^{\frac{1}{\sqrt{1-\frac{\alpha_{t}}{\alpha_{c}}}}},
$$

showing that the infrared scale $\mu^{2}$ is directly proportional to the ultraviolet scale $\Lambda^{2}$, which is the unique scale in the theory. As a result the top quark mass $m_{t} \sim A_{t} \mu$ is generated by the spontaneous symmetry breaking. Due to the absence of the four-fermion coupling in the gap-equation (15) for the bottom quark, there exists only the trivial solution, i.e., the self-energy function $\Sigma_{b}(p)$ of the bottom quark in the chiral limit and small QED gauge coupling is identically zero,

$$
A_{b}=0 \quad \Sigma_{b}(p) \equiv 0 .
$$

The bottom quark remains massless because its Schwinger-Dyson equation is homogeneous [5].

Once the system undergoes the spontaneous symmetry breaking, there are Goldstone bosons [1, [6] above infinitely degenerate ground states and the value of the infrared scale $\mu$ totally depends on the couplings $g$ and $\alpha_{t}$ chosen. 
All "Yukawa coupling" $A$ 's are independent parameters that do not enter the gap equations. In order to achieve the right weak scale $(\mu \sim 250 \mathrm{GeV})$, we need an extreme fine-tuning of the four-fermion coupling $G$,

$$
\left(\frac{\mu}{\Lambda}\right)^{2}=\frac{\alpha_{t}}{4 \alpha_{c}} \frac{G+\frac{\alpha_{t}}{\alpha_{c}}-4}{G-\frac{\alpha_{t}}{\alpha_{c}}} \sim 10^{-34} ; \quad G \equiv \frac{N_{c} G_{\circ} \Lambda^{2}}{2 \pi^{2}}
$$

where the cutoff is assumed at the Planck scale and the gauge coupling $\frac{\alpha_{t}}{\alpha_{c}} \ll 1$. The critical value is thus given by $G+\frac{\alpha_{t}}{\alpha_{c}}=4$. As already noted, this fine-tuning is due to the existence of the quadratic divergence in the gapequation (20) for the self-energy function. The fact that a totally unnatural fine-tuning is forced in order to not only obtain non-perturbative dynamical symmetry breaking $\mu \neq 0$, but also remove the quadratic divergence to reach the low-energy limit $\mu \ll \Lambda$ signals a non-trivial structure of the ground states in the high-energy region.

\section{The vector-like phenomenon and three-fermion states}

Due to the underlying physics at the cutoff, beside the dimension-6 operator (11), the effective lagrangian could contain other high dimensional operators, which obey the gauge symmetries of the Standard Model. The effective coupling of these operators could be momentum-dependent. When the effective couplings are larger than a certain threshold value $\epsilon$, three-fermion Weyl states and other composite states with appropriate quantum numbers are bound, and the mass spectra and the 1PI vertices are vector-like, consistently with chiral gauge symmetries of the Standard Model. We will not enter into the details of this phenomenon that is described in ref. [7]. In this article, instead, we wish to reconsider the Schwinger-Dyson equations (2, 3) for the self-energy functions $\Sigma_{t}(p)$ and $\Sigma_{b}(p)$ of the top and bottom quarks by taking into account the possible relevant $1 \mathrm{PI}$ vertex functions arising in the high-energy region. Thus, inspired by such vector-like phenomenon in the high energy region, we postulate beyond a certain energy scale $\epsilon$, larger than the weak scale,

- the existence of right-handed three-fermion Weyl states with the chiral gauge quantum numbers of the weak $S U_{L}(2)$ group. These states combine with elementary Weyl fermions to form massive Dirac fermions; 
- the vector-like character of the coupling vertex between these composite Dirac fermions and the $W^{ \pm}$gauge boson.

We are now going to explicitly discuss these two assumptions within the third quark family in the high energy region. We assume there is an intermediate energy-threshold $\epsilon$ between the weak scale $(\mu \sim 250 \mathrm{GeV})$ and the cutoff $\Lambda$,

$$
250 \mathrm{GeV}<\epsilon<\Lambda,
$$

above which the effective couplings of high dimensional operators are strong enough to form the three-fermion bound states of the third quark family that are given by,

$$
T_{R} \sim\left(\bar{t}_{R} \cdot t_{L}\right) t_{R}, \quad B_{R} \sim\left(\bar{b}_{R} \cdot b_{L}\right) b_{R},
$$

which are right-handed Weyl fermions with the appropriate gauge quantum number of $S U_{L}(2)$. On the other hand, as the effective couplings decrease, these right-handed three-fermion bound states disappear (turning to unbound three fermion states ) at the threshold $\epsilon$ (21), to reproduce the parity-violating gauge coupling of the low-energy phenomenology,

In the high energy region, the right-handed fermion state comprises the elementary state $t_{R}\left(b_{R}\right)$ and the composite state $T_{R}\left(B_{R}\right)$ :

$$
\Psi_{R}^{t}=\left\{t_{R}, T_{R}\right\} ; \quad \Psi_{R}^{b}=\left\{b_{R}, B_{R}\right\} .
$$

The composite Dirac particles are then given,

$$
\Psi_{D}^{t}=\left\{t_{L}, \Psi_{R}^{t}\right\} ; \quad \Psi_{D}^{b}=\left\{b_{L}, \Psi_{R}^{b}\right\}
$$

which couple to the $W^{ \pm}$-gauge boson in a vector-like manner. Thus the effective gauge coupling of $W^{ \pm}$-bosons to quarks in the high-energy region becomes vector-like. For the quark- $W^{ \pm}$vertex one may write:

$$
\begin{aligned}
\Gamma_{\mu}^{w}(q) & =i g_{w}(q) \gamma_{\mu}\left(P_{L}+f(q)\right) \\
f(q) & \neq 0 \text { for } q>\epsilon,
\end{aligned}
$$

where the CKM-matrix $[8]$ is set to be identity and $g_{w}(q)$ is a renormalized coupling constant. Below the energy threshold (21) where the three-fermion states dissolve into their constituents, we assume that the vertex function $f(q)$ vanishes, i.e.

$$
\left.f(q)\right|_{q \rightarrow \epsilon}=0 .
$$


The scenario of the vector-like phenomenon above the intermediate scale (21) is reminiscent of "left-right" symmetric extensions of the Standard model 9].

The self-energy functions $\Sigma_{t, b}(p)$ in the high-energy region $(p \gg 1)$ should be defined as the truncated two-point functions of the elementary left-handed fields $\left(t_{L}, b_{L}\right)$ and the right-handed mixing states $\left(\Psi_{R}^{t}, \Psi_{R}^{b}\right)(23)$. Thus, we find that the $W^{ \pm}$bosons give the following contributions to the RHS of the Schwinger-Dyson equations (2, 3) in the high-energy region $\left(p^{\prime} \geq \epsilon\right)$ :

$$
\begin{aligned}
W_{b}(p) & =\int_{\left|p^{\prime}\right| \geq \epsilon}^{\Lambda} \frac{Q_{w}^{2}}{\left(p-p^{\prime}\right)^{2}+M_{w}^{2}} \frac{\Sigma_{t}\left(p^{2}\right)}{p^{\prime 2}+\Sigma_{t}^{2}\left(p^{\prime 2}\right)} ; \\
W_{t}(p) & =\int_{\left|p^{\prime}\right| \geq \epsilon}^{\Lambda} \frac{Q_{w}^{2}}{\left(p-p^{\prime}\right)^{2}+M_{w}^{2}} \frac{\Sigma_{b}\left(p^{\prime 2}\right)}{p^{\prime 2}+\Sigma_{b}^{2}\left(p^{\prime 2}\right)},
\end{aligned}
$$

where $Q_{w}$ is the weak charge (25), and the integration of the internal momentum $p^{\prime}$ starts from the intermediate threshold $\epsilon$ to the cut-off $\Lambda$.

Due to the properties of the right-handed composite states $T_{R}$ and $B_{R}$ (22) that carry the appropriate quantum numbers and couple to the $W^{ \pm}$ gauge bosons, the fermionic self-energy functions $\Sigma_{t, b}(p)$ do not violate the chiral gauge symmetries of the Standard model in the high-energy region $(p>\epsilon)$, where the spontaneous symmetry breaking is soft and thus irrelevant. Thus the self-energy functions $\Sigma_{t, b}(p)(p>\epsilon)$ can be non-vanishing without violating the $S U_{L}(2)$ chiral gauge symmetry, and the corresponding Ward identity relates the self-energy functions $\Sigma_{t, b}(p)$ to the vector-like vertex function $f(q)(25)$.

\section{Solution to the coupled Schwinger-Dyson equations}

The $W$-bosons perturbatively contribute to the original Schwinger-Dyson equations for the top quark (2) and the bottom quark (3) respectively. One can see that eq.(28) mixes the Schwinger-Dyson equations for the fermionic self-energy functions of different charge sectors. As a consequence, eqs. (2, 3) are no longer independent equations, instead, they are perturbatively coupled together:

$$
\begin{aligned}
& \Sigma_{t}\left(p^{2}\right)=-\frac{G_{\circ}}{2}\langle\bar{t} t\rangle+W_{t}(p)+3 Q^{2} \int_{p^{\prime}}^{\Lambda} \frac{1}{\left(p-p^{\prime}\right)^{2}} \frac{\Sigma_{t}\left(p^{\prime 2}\right)}{p^{\prime 2}+\Sigma_{t}^{2}\left(p^{\prime 2}\right)} \\
& \Sigma_{b}\left(p^{2}\right)=W_{b}(p)+\frac{3 Q^{2}}{4} \int_{p^{\prime}}^{\Lambda} \frac{1}{\left(p-p^{\prime}\right)^{2}} \frac{\Sigma_{b}\left(p^{\prime 2}\right)}{p^{\prime 2}+\Sigma_{b}^{2}\left(p^{\prime 2}\right)}
\end{aligned}
$$


where the bare masses $m$ are set to zero. For $p \gg 1$ and $\left|p^{\prime}\right| \geq \epsilon \gg 1$, we approximate the W's contributions (28) to be,

$$
W_{b, t}(p)=\int_{\left|p^{\prime}\right| \geq \epsilon}^{\Lambda} \frac{Q_{w}^{2}}{\left(p-p^{\prime}\right)^{2}+M_{w}^{2}} \frac{\Sigma_{t, b}\left(p^{\prime 2}\right)}{p^{\prime 2}+\Sigma_{t, b}^{2}\left(p^{\prime 2}\right)} \simeq \alpha_{w} \Sigma_{t, b}(\Lambda),
$$

where $\alpha_{w}$ is an undetermined constant.

Analogously to eqs.(田, 6), in the ultraviolet region $\left(x=p^{2} \gg 1\right)$, the integral eqs. 29. 30) can be converted to the following boundary value problems:

$$
\begin{aligned}
\frac{d}{d x}\left(x^{2} \Sigma_{t}^{\prime}(x)\right)+\frac{\alpha_{t}}{4 \alpha_{c}} \Sigma_{t}(x) & =0, \\
(1+g) \Lambda^{2} \Sigma_{t}^{\prime}\left(\Lambda^{2}\right)+\Sigma_{t}\left(\Lambda^{2}\right) & =\alpha_{w} \Sigma_{b}(\Lambda),
\end{aligned}
$$

and

$$
\begin{aligned}
\frac{d}{d x}\left(x^{2} \Sigma_{b}^{\prime}(x)\right)+\frac{\alpha_{t}}{16 \alpha_{c}} \Sigma_{b}(x) & =0, \\
\Lambda^{2} \Sigma_{b}^{\prime}\left(\Lambda^{2}\right)+\Sigma_{b}\left(\Lambda^{2}\right) & =\alpha_{w} \Sigma_{t}(\Lambda) .
\end{aligned}
$$

These differential equations are the same as before, except for the fact that now the ultraviolet boundary conditions are coupled. Thus, substituting the known generic solutions (9:10) for $x \gg 1$ into the boundary conditions (33) and (35), we obtain the self-consistent gap-equations for $\Sigma_{t}(\Lambda)$ and $\Sigma_{b}(\Lambda)$,

$$
\begin{aligned}
\alpha_{w} \Sigma_{b}(\Lambda) & =\frac{A_{t} \mu^{2}}{2 \Lambda}\left[(1-g) \sinh \theta+(1+g) \sqrt{1-\frac{\alpha_{t}}{\alpha_{c}}} \cosh \theta\right] \\
\alpha_{w} \Sigma_{t}(\Lambda) & =\frac{A_{b} \mu^{2}}{2 \Lambda}\left[\sinh \theta^{\prime}+\sqrt{1-\frac{\alpha_{t}}{4 \alpha_{c}}} \cosh \theta^{\prime}\right],
\end{aligned}
$$

which differ in an essential way from the self-consistent gap-equations (11, 12) for the decoupled system.

The first conclusion that one can derive from these gap-equations is that the self-energy function of the bottom quark must be non-trivial

$$
\Sigma_{b}(\Lambda) \neq 0 ; \text { if } \Sigma_{t}(\Lambda) \neq 0,
$$

where the $\Sigma_{t}(\Lambda)$ is generated by the spontaneous symmetry breaking for large value of the four-fermion coupling $G_{\circ}$. The bottom quark mass is generated 
by the explicit symmetry breaking, due to the top quark mass. There is another trivial-solution:

$$
\Sigma_{b}(\Lambda)=0 ; \text { and } \Sigma_{t}(\Lambda)=0
$$

These solutions are not energetically favorable for large coupling $G_{\circ}$.

We now turn to find the solution of the gap-equations (36,37) in the low-energy limit $(\mu \ll \Lambda)$. These equations become

$$
\begin{aligned}
& \alpha_{w} \Sigma_{b}(\Lambda)=\frac{A_{t} \mu}{4}\left(\frac{\mu}{\Lambda}\right)^{1-\sqrt{1-\frac{\alpha_{t}}{\alpha_{c}}}}\left[1-g+(1+g) \sqrt{1-\frac{\alpha_{t}}{\alpha_{c}}}\right] \\
& \alpha_{w} \Sigma_{t}(\Lambda)=\frac{A_{b} \mu}{4}\left(\frac{\mu}{\Lambda}\right)^{1-\sqrt{1-\frac{\alpha_{t}}{4 \alpha_{c}}}}\left[1+\sqrt{1-\frac{\alpha_{t}}{4 \alpha_{c}}}\right] .
\end{aligned}
$$

Taking the ratio of these two equations, we obtain

$$
\frac{\Sigma_{b}(\Lambda)}{\Sigma_{t}(\Lambda)}=\left(\frac{A_{t} \mu}{A_{b} \mu}\right)\left(\frac{\mu}{\Lambda}\right)^{\frac{3 \alpha_{t}}{8 \alpha_{c}}}\left[\frac{1-g+(1+g) \sqrt{1-\frac{\alpha_{t}}{\alpha_{c}}}}{1+\sqrt{1-\frac{\alpha_{t}}{4 \alpha_{c}}}}\right] .
$$

Considering the effect of renormalization on the gauge interactions (QCD, QED) and fermionic mass operators, we obtain

$$
\frac{\Sigma_{b}(\Lambda)}{\Sigma_{t}(\Lambda)}=\frac{\Sigma_{b}(\mu)}{\Sigma_{t}(\mu)}\left(\frac{\alpha_{t}(\Lambda)}{\alpha_{t}(\mu)}\right)^{\frac{9}{20 N_{F}}}
$$

where $N_{F}$ is the number of quark flavours. We can appropriately choose constants $A_{t}$ and $A_{b}$ so that the masses of the top and bottom quarks at the infrared scale $\mu$ are given by,

$$
\Sigma_{t}(\mu) \simeq A_{t} \mu ; \quad \Sigma_{b}(\mu) \simeq-A_{b} \mu
$$

where we attribute the fermion masses $m_{t, b}$ an opposite sign. Thus, we get the following relationship

$$
\left(\frac{\Sigma_{b}(\mu)}{\Sigma_{t}(\mu)}\right)^{2}\left(\frac{\alpha_{t}(\Lambda)}{\alpha_{t}(\mu)}\right)^{\frac{9}{20 N_{F}}}\left(1+\frac{3 \alpha_{t}(\Lambda)}{8 \alpha_{c}} \ln \frac{\Lambda}{\mu}\right)=\frac{G+\frac{\alpha_{t}(\Lambda)}{\alpha_{c}}-4}{4-\frac{\alpha_{t}(\Lambda)}{4 \alpha_{c}}} .
$$


This is a gap equation relating the masses $\Sigma_{t, b}(\mu)$ of the top and bottom quarks and the couplings $G, \alpha_{t}$, similar to the gap-equations (16,17) obtained in the case of explicit symmetry breakings.

\section{Discussions and remarks}

As remarked after eq.(20), the problem of unnatural fine-tuning arises from the fact that we must simultaneously arrange the couplings $G$ and $\alpha_{t}$ in such a way that the the quadratic divergence in the cut-off $\Lambda$ gets cancelled and in addition the soft spontaneous symmetry breaking is nontrivial yielding $\mu \neq 0, \mu \ll \Lambda$. On the other hand, the coupled gap-equations (40,41) have eq.(45) as their implication, showing that we can dispose of the the cancellation of the quadratic divergence $\Lambda^{2}$ and of the non-trivial soft spontaneous symmetry breaking in an independent way. Indeed we may assume that $G$ goes exactly to the fixed point, i.e.

$$
G \rightarrow 4+0^{+}
$$

where the $\Lambda^{2}$ is exactly cancelled and the soft spontaneous symmetry breaking $(\mu \neq 0)$ gets in touch with the cut-off $\Lambda$ only logarithmically through the relationship:

$$
\left(\frac{\Sigma_{b}(\mu)}{\Sigma_{t}(\mu)}\right)^{2}\left(\frac{\alpha_{t}(\Lambda)}{\alpha_{t}(\mu)}\right)^{\frac{9}{20 N_{F}}}\left(1+\frac{3 \alpha_{t}(\Lambda)}{8 \alpha_{c}} \ln \frac{\Lambda}{\mu}\right) \simeq \frac{\frac{\alpha_{t}(\Lambda)}{\alpha_{c}}}{4-\frac{\alpha_{t}(\Lambda)}{4 \alpha_{c}}}
$$

obtained from eq(45) through (46). In eq.(47), no fine-tuning of the electric coupling $\alpha$ is needed to have $\mu \ll \Lambda$.

The reason for this appearing result is that we are approaching the physical fixed point, i.e. $\left(G, \alpha_{t}\right) \rightarrow\left(4, \frac{4}{9} \frac{1}{137}\right)$, from the spontaneous symmetry broken phase. At this fixed point, the effective dimensions of the four-fermion operator (11) becomes 4 [4] and the $\Lambda$-dependence is only logarithmic. Note that this is true for both the the decoupled gap-equation (20) and coupled gap-equation (45). However in the decoupled case we must fine-tune the approach to the fixed point up to an unreasonable precision $O\left(\frac{\mu^{2}}{\Lambda^{2}}\right)$, while in the coupled case we must demand that $G \rightarrow 4$ only up to $o\left(\frac{m_{b}^{2}}{m_{t}^{2}}\right)$. If we are willing to accept that, for some general principle still to be studied, $G=4+0^{+}$(or else $\left.G=4+o\left(\frac{m_{b}^{2}}{m_{t}^{2}}\right)\right)$, we obtain an interesting prediction 
on the mass ratio $\left(\frac{m_{b}}{m_{t}}\right)$. In fact, taking the value $\alpha^{-1}\left(M_{Z}\right) \simeq 12810$ and $\Lambda=10^{19} \mathrm{GeV}$, by renormalization group equation we obtain $\alpha^{-1}(\Lambda) \simeq 103$. From equation (47), one can make the estimate

$$
\left(\frac{m_{b}\left(M_{Z}\right)}{m_{t}\left(M_{Z}\right)}\right)^{2} \simeq 0.93 \frac{\alpha_{t}}{3 \pi} \quad \alpha_{t}=\frac{1}{103}
$$

which is in consistent with experiment values.

We wish to thank Profs. Y. Nambu, E. Akhmedov, M. Lindner, H.Q. Zheng for stimulating discussions.

\section{References}

[1] Y. Nambu and G. Jona-Lasinio, Phys. Rev. 122 (1961) 345.

[2] W.A. Bardeen, C. T. Hill and M. Lindner Phys. Rev. D41 (1990) 1647.

[3] G. Preparata and S.-S. Xue, Phys. Lett. B337 (1996) 124, Il Nuovo Cimento A109 (1996) 137. S.-S. Xue, Nucl. Phys. B47 (Proc. Suppl.) (1996) 583.

[4] W.A. Bardeen, C.N. Leung, and S.T. Love, Nucl. Phys. B273 (1986) 649; ibid B323 (1989) 493.

A. Kocić, S. Hands, B. Kogut and E. Dagotto, Nucl. Phys. B347 (1990) 217.

[5] T. Maskawa and H. Nakajima, Prog. Theor. Phys. 52 (1974) 1326; ibid 54 (1975) 860;

R. Fukuda and T. Kugo, Nucl. Phys. B117 (1976) 250.

[6] J. Goldstone, Nuovo Cimento 19 (1961) 154.

[7] S.-S. Xue, Phys. Lett. B381 (1996) 277 and Nucl. Phys. B486 (1997) 282; G. Preparata and S.-S. Xue Phys. Lett. B (1997).

[8] N. Cabibbo, Phys. Rev. Lett. 10 (1963) 531;

M. Kobayashi and M. Maskawa, Prog. Theor. Phys. 49 (1973) 652. 
[9] J.C. Pati and A. Salam, Pyhs. Rev. D10 (1975) 275;

R.N. Mohapatra and P.C. Pati, ibid D11 (1975) 566; D11 (1975) 2558; G. Senjanović and R.N. Mohopatra, Pyhs. Rev. D12 (1975) 1502;

R.N. Mohopatra and G. Senjanović, Pyhs. Rev. D23 (1981) 165 and Pyhs. Rev. Lett. 44 (1980) 912;

E. Akhmedov, M. Lindner, E. Schnapka, and J.W.F. Valle, Pyhs. Rev. D53 (1996) 2752; Pyhs. Lett. B368 (1996) 270.

[10] The Review of Particle Properties, Phys. Rev. D50 (1994) 1173. 